CUBO A Mathematical Journal

Vol.12, Nํㅡㄹ, (123-126). June 2010

\title{
A note on generalized topological spaces and preorder
}

\author{
SAEID JAFARI \\ College of Vestsjaelland South, \\ Herrestraede 11, \\ 4200 Slagelse, Denmark, \\ email: jafari@stofanet.dk \\ RAJA MOHAMMAD LATIF \\ Department of Mathematics and Statistics, \\ King Fahd University of Petroleum and Minerals, \\ Dhahran 31261, Saudi Arabia. \\ email: raja@kfupm.edu.sa \\ and \\ Seithuti P. MoshokoA \\ Department of Mathematical Sciences, \\ University of South Africa, \\ P.O. Box 392, Pretoria 0003, South Africa. \\ email: moshosp@unisa.ac.za
}

\begin{abstract}
This paper deals with the notion of continuity in ordered generalized topological spaces. We also characterize general topological spaces satisfying the Hausdorff property via the graph of the preorder assigned to the general topological space.

\section{RESUMEN}

Este artículo trata de continuidad en espacios topológicos ordenados. También caracterizamos espacios topológicos generalizados satisfaciendo la propiedad de Hausdorff vía el grafo designado de un espacio topológico general preordenado.
\end{abstract}


Key words and phrases: Topological Spaces, Hausdorff, preorder.

AMS Subject Classification (2000): 54D10

\section{Introduction}

By a generalized topology ( briefly GT) on $X$ [1], we mean a subset $\mu$ of the power set exp X, of $X$ such that $\emptyset \in \mu$ and arbitrary union of elements of $\mu$ belongs to $\mu$. The elements of $\mu$ are said to be $\mu$-open, their compliments are said to be $\mu$-closed. Clearly, each topology is a GT, but not conversely. As a generalization of the concept of a Hausdorff topology, we shall say that a GT, written $(X, \mu)$ is Hausdorff if for distinct points $x$ and $y$ in $X$ there exists disjoint $\mu$-open sets $U$ and $V$ containing $x$ and $y$, respectively. By $(X, \mu, \leq)$ we mean a GT together with a preorder. Let $A$ be a subset of $X$, let $i(A)=\cup_{x \in A} x^{\uparrow}$, where $x^{\uparrow}=\{y \in X: x \leq y\}$. Let $d(A)=\cup_{x \in A} x^{\downarrow}$, where $x^{\downarrow}=\{y \in X: y \leq x\}$. Clearly, we have $A \subseteq i(A)$ and $A \subseteq d(A)$ for all $A \subseteq X$. We say that a subset $A$ of $X$ is increasing (decreasing) if $i(A)=A(A=d(A))$. If the preorder is discrete, then $(X, \mu, \leq)$ is just a GT, as in the literature, see for example [1] [2] . We shall simply write OGT, for a ordered generalized topological space $(X, \mu, \leq)$. Now let $G=\{(x, y): x \leq y\}$. Then $G$ is a subset of $X \times X$, we shall refer to $G$ as the graph of the preorder. We shall say that a subset $G$ is closed if $X \times X-G$ is open in the product generalized topology, denoted by $\mu \times \mu$. That is, a subset $B$ of $X \times X$ is $(\mu \times \mu)$-open if for every $(x, y) \in B$ there exist $\mu$-open sets $U$ and $V$, such that $(x, y) \in U \times V \subset B$, where $U$ and $V$ contain $x$ and $y$, respectively. We shall say that the preorder on $X$ is $(\mu \times \mu)$-closed if its graph $G$ is $(\mu \times \mu)$-closed in $X \times X$.

\section{Results}

Let $A$ be a subset of $X$. We denote the $\mu$-closure of $A$ by $c_{\mu} A$, and recall:

Lemma 2.1. [2] If $\mu$ is a $G T$ on $X, A \subset X, x \in X$, then $x \in c_{\mu} A$ if and only if $x \in U \in \mu$ implies $U \cap A \neq \emptyset$.

Proof. Define $B=\{x \in X: x \in U \in \mu \Rightarrow U \cap A \neq \emptyset\}$. Clearly $A \subset B$. Further $B$ is $\mu$-closed, that is $X-B \in \mu$ because $x \in X-B$ if and only if there is $U_{x} \in \mu$ such that $x \in U_{x}$ and $U_{x} \cap A \neq \emptyset$ and then $y \in U_{x}$ implies that $y \in X-B$ so that $X-B=\cup_{x \in X-B} U_{x} \in \mu$. Finally, if $A \subset Q$ and $Q$ is $\mu$-closed, then $X-Q \in \mu$ and $(X-Q) \cap A=\emptyset$ so that $X-Q \subset X-B, B \subset Q$. Therefore $B$ is the smallest $\mu$-closed set containing $A$, i.e. $B=c_{\mu} A$.

Clearly, for a subset $A$ of $(X, \mu)$ we have $A \subset c_{\mu} A$. Also, $c_{\mu} A$ is $\mu$-closed. To see this just note that the $\mu$-closure of $A$ is the intersection of all $\mu$-closed supersets of $A$ in $X$. Therefore, the complement of the $\mu$-closure of $A$ is $\mu$-open, being an arbitrary union of members of $\mu$.

Example 2.2. Let $X=\{1,2,3\}$ and define $\mu=\{\emptyset,\{1,2\},\{2,3\},\{1,2,3\}\}$. Then $(X, \mu, \leq)$ is an $O G T$, where the preorder is induced from $N$ but it is not an ordered topological space. Also, for the subset $A=\{3\}$ we have $c_{\mu} A=A$.

We shall need the following: 
Theorem 2.3. Let $\mu$ be a $G T$ on $X$, if $A \subseteq B$ in $X$, then $c_{\mu} A \subseteq c_{\mu} B$.

Proof. Let $(X, \mu)$ be a GT, and $A$ and $B$ be subsets of $X$ such that $A \subseteq B$. If $x \in c_{\mu} A$, then by Lemma 2.1, there exists $U_{x} \in \mu$ containing $x$ such that $U_{x} \cap A \neq \emptyset$. Since $A \subseteq B$, we have $U_{x} \cap B \neq \emptyset$. Therefore $x \in c_{\mu} B$. This shows that $c_{\mu} A \subseteq c_{\mu} B$.

We will say that $(X, \mu, \leq)$ is $\mu$-continuous ( $\mu$-anticontinuous) is and only if for each $\mu$-open subset $G$ ( $\mu$-closed subset $F) i(G)$ and $d(G)$ are $\mu$-open $(i(F)$ and $d(F)$ are $\mu$-closed). Also, we say that $(X, \mu, \leq)$ is bicontinuous if it is both continuous and anti-continuous.

Example 2.4. Let the $O G T(X, \mu, \leq)$ be that of Example 2.1. Then for every $\mu$-open set $A$ and $\mu$-closed set $B$ in $X$, the sets: $i(A)$ and $d(A)$ are $\mu$-open and the sets $i(B)$ and $d(B)$ are $\mu$-closed. It follows that the $O G T(X, \mu, \leq)$ is bicontinuous.

Theorem 2.5. The space $(X, \mu, \leq)$ is anti-continuous if and only if

(i) $c_{\mu}(i(A)) \subseteq i\left(c_{\mu}(A)\right)$ and $c_{\mu} d(A) \subseteq d\left(c_{\mu} A\right)$ for every $A \subseteq X$.

(ii) $(X, \mu, \leq)$ is continuous if and only if $i\left(c_{\mu} A\right) \subseteq c_{\mu}(i(A))$ and $d\left(c_{\mu} A\right) \subseteq c_{\mu} d(A)$ for every $A \subseteq X$.

(iii) $(X, \mu, \leq)$ is bicontinuous if and only if $i\left(c_{\mu} A\right)=c_{\mu}(i(A))$ and $d\left(c_{\mu} A\right)=c_{\mu} d(A)$ for every $A \subseteq X$.

Proof. (i) Suppose that $(X, \mu, \leq)$ is anticontinuous and $A$ be a subset of $X$. Note that $A \subseteq c_{\mu} A$, and therefore $i(A) \subseteq i\left(c_{\mu} A\right)$. We apply Theorem 2.3, to obtain $c_{\mu}(i(A)) \subseteq c_{\mu}\left(i\left(c_{\mu} A\right)\right)$. Note that $i\left(c_{\mu} A\right)$ is $\mu$-closed as $(X, \mu, \leq)$ is anticontinuous, we then have $c_{\mu}\left(i\left(c_{\mu} A\right)\right)=i\left(c_{\mu} A\right)$. Hence $c_{\mu}(i(A)) \subseteq$ $i\left(c_{\mu}(A)\right)$. Dually, we can show $c_{\mu} d(A) \subseteq d\left(c_{\mu} A\right)$. Conversely, suppose that $c_{\mu}(i(A)) \subseteq i\left(c_{\mu}(A)\right)$ holds for all $A \subseteq X$. Let $F$ be a $\mu$-closed subset of $X$. First, we note that $i(F) \subseteq c_{\mu} i(F)$ this holds even when $F$ is not necessarily $\mu$-closed. On the other hand, let $F$ be $\mu$-closed and $c_{\mu}(i(F)) \subseteq i\left(c_{\mu}(F)\right)$ holds. We have $c_{\mu} i(F) \subseteq i(F)$. Hence $c_{\mu} i(F)=i(F)$. This shows that $i(F)$ is $\mu$-closed. Dually, $d(F)$ is $\mu$-closed.

(ii) Let $(X, \mu, \leq)$ be continuous and $A$ be a subset of $X$. Then $c_{\mu} i(A)$ is $\mu$-closed, so is that $X-c_{\mu} i(A)$ is $\mu$-open. Hence, $X-d\left(X-c_{\mu} i(A)\right)$ is $\mu$-closed. Note that $X-d\left(X-c_{\mu} i(A)\right)$ is increasing and contains $A$. It follows that $c_{\mu} A \subseteq X-d\left(X-c_{\mu} i(A)\right)$. Since $A \subseteq d(A)$ for all $A \subseteq X$, we have

$$
X-c_{\mu} i(A) \subseteq d\left(X-c_{\mu} i(A)\right) .
$$

This implies that $X-d\left(X-c_{\mu} i(A)\right) \subseteq c_{\mu} i(A)$. Therefore,

$$
c_{\mu} i(A)=X-d\left(X-c_{\mu} i(A)\right) .
$$

Hence $i\left(c_{\mu} A\right) \subseteq c_{\mu} i(A)$. Dually, we have $d\left(c_{\mu} A\right) \subseteq c_{\mu} d(A)$ Conversely, suppose that $i\left(c_{\mu} A\right) \subseteq c_{\mu} i(A)$ and $d\left(c_{\mu} A\right) \subseteq c_{\mu} d(A)$ holds for all $A \subseteq X$. Let $G$ be a $\mu$-open subset of $X$. Then $X-G$ is $\mu$-closed and $c_{\mu}(X-i(G)) \subseteq X-G$, hence $G \subset X-c_{\mu}(X-i(G))$. It follows that

$$
d\left(c_{\mu}(X-i(G))\right) \subseteq_{\mu} d(X-i(G))=c_{\mu}(X-i(G))
$$

so that $c_{\mu}(X-i(G))$ is decreasing. Thus $i(G) \subset X-c_{\mu}(X-i(G))$. On the other hand, it is clear that $X-i(G) \subseteq c_{\mu}(X-i(G))$ and hence

$$
X-c_{\mu}(X-i(G)) \subseteq i(G) .
$$


Consequently, $i(G)=X-c_{\mu}(X-i(G))$ is $\mu$-open. Dually, $d(G)$ is $\mu$-closed.

(iii) Suppose that $(X, \mu, \leq)$ is bicontinuous. Then $(X, \mu, \leq)$ is both anticontinuous and continuous. So by $(i)$ we have $c_{\mu}(i(A)) \subseteq i\left(c_{\mu}(A)\right)$ and $c_{\mu} d(A) \subseteq d\left(c_{\mu} A\right)$ for every $A \subseteq X$. Also by $(i i)$, we have $i\left(c_{\mu} A\right) \subseteq c_{\mu}(i(A))$ and $d\left(c_{\mu} A\right) \subseteq c_{\mu} d(A)$. Together, we get $i\left(c_{\mu} A\right)=c_{\mu}(i(A))$ and $d\left(c_{\mu} A\right)=c_{\mu} d(A)$ for every $A \subseteq X$. The converse is clear from $(i)$ and $(i i)$.

Theorem 2.6. Let $(X, \mu, \leq)$ be an $O G T$. Then the preorder $\leq$ is closed if and only if, for every two points $a, b \in X$ such that $a \leq b$ is false, it is possible to determine a increasing $\mu$-open set $U$ that contains $a$ and a decreasing set $\mu$-open set $V$ containing $b$, such that $U \cap V=\emptyset$.

Proof. Let $a, b \in$ such that $a \leq b$ is false, that is, $(a, b) \notin G$. Since $G$ is $(\mu \times \mu)$-closed, we have that $X \times X-G$ is $(\mu \times \mu)$-open and $(a, b) \in X \times X-G$. Now find $\mu$-open sets $\dot{U}$ and $\dot{V}$ containing $a$ and $b$, respectively such that $U^{\prime} \times V^{\prime} \cap G=\emptyset$. Now if $x \in \dot{U}^{\prime}$ and $y \in \dot{V}$, then $x \leq y$ is false. We set $U=i(\dot{U})$ and $V=d(\dot{V})$. Now, $\dot{U} \subset U$ and $\dot{V} \subset V$. Then $U$ is $\mu$-open increasing and contains $a$, and $V$ is $\mu$-open decreasing and contains $b$. Next, we show that $U \cap V=\emptyset$. Suppose that $U \cap V \neq \emptyset$, let $z \in U \cap V$. Since $z \in U$, there exists a point $x \in U^{\prime}$ such that $x \leq z$. Also, $z \in V$ gives a point $y \in \dot{V}$, such that $z \leq y$. Hence, $x \leq y$ follows from the fact that the " $\leq$ " is a preorder on $X$. On the other hand $x \in U^{\prime}$ and $y \in V^{\prime}$ implies that $x \leq y$ is false. So we arrive at a contradiction. We conclude that $U \cap V=\emptyset$. Conversely, assume that $G$ is not closed, then find $(a, b)$ in the $\mu$-closure of $G$, such that $(a, b) \notin G$. Then consider the sets $U$ and $V$ containing $a$ and $b$, increasing and decreasing, respectively. Then $U \cap V$ contains $(a, b)$ and satisfy $U \times V \cap G \neq \emptyset$. Observe that $U \times V$ is $(\mu \times \mu)$-open. So, find $u \in U$ and $v \in V$ such that $(u, v) \in G$ and $u \leq v$. From $u \in U$ and $u \leq v$ results that $v \in U$, since $U$ is increasing; thus $V$ belongs to both $U$ and $V$, that is $U$ and $V$ are not disjoint.

Theorem 2.7. Let $(X, \mu, \leq)$ be an OGT. If the preorder $\leq$ is closed, then for every point $a \in X$, the sets $d(a)$ and $i(a)$ are $\mu$-closed.

Proof. Suppose that the preorder is closed that is its graph $G$ is closed. Let $a \in X$. If $b \in X-i(a)$, then $a \leq b$ is false. By Theorem 2.6, there exists an increasing $\mu$-open set $U$ containing $a$ and a decreasing $\mu$-open set $V$ containing $b$ such that $U$ and $V$ are disjoint. Now $i(a) \subset U$ implies that

$$
W \cap i(a) \neq \emptyset
$$

this shows that $i(a)$ is closed.

Acknowledgement. The second author is highly and greatly indebted to the King Fahd University of Petroleum and Minerals, for providing necessary research facilities during the preparation of this paper. The third author has been supported by the South African National Research Foundation.

Received: January 2009. Revised: March 2009.

\section{References}

[1] A. Csaszar, Generalized topology, generalized continuity, Acta Math. Hungar., 96 (2oo2), 351357.

[2] A. Csaszar, $\delta$ - and $\theta$ - modifications of generalized topologies, Acta. Math. Hungar., to appear. 